\title{
Evaluation of a wearable device to determine cardiorespiratory parameters from surface diaphragm electromyography
}

\author{
Magda Ràfols-de-Urquía, Luis Estrada, Senior Member, IEEE, Josep Estévez-Piorno, Leonardo \\ Sarlabous, Raimon Jané, Senior Member, IEEE and Abel Torres, Member, IEEE
}

\begin{abstract}
Using wearable devices in clinical routines could reduce healthcare costs and improve the quality of assessment in patients with chronic respiratory diseases. The purpose of this study is to evaluate the capability of a Shimmer 3 wearable device device to extract reliable cardiorespiratory parameters from surface diaphragm electromyography (EMGdi). Twenty healthy volunteers underwent an incremental load respiratory test whilst EMGdi was recorded with a Shimmer3 wearable device (EMGdiw). Simultaneously, a second EMGdi (EMGdiL), the inspiratory mouth pressure (Pmouth) and the lead-I electrocardiogram (ECG) were recorded via a standard wired laboratory acquisition system. Different cardiorespiratory parameters have been extracted from both $\mathrm{EMGdi}_{W}$ and $\mathrm{EMGdi}_{L}$ signals.: heart rate, respiratory rate, respiratory muscle activity and mean frequency of EMGdi signals. Alongside these, similar parameters were also extracted from reference signals (Pmouth and ECG). High correlations were found between the data extracted from the EMGdiw and the reference signal data: heart rate $(R=0.947)$, respiratory rate $(R=0.940)$, respiratory muscle activity $(R=0.877)$, and mean frequency $(R=$ 0.895). Moreover, similar increments in EMGdiw and EMGdiL activity were observed when Pmouth was raised, enabling the study of respiratory muscle activation. In summary, the Shimmer3 device is a promising and cost-effective solution for ambulatory monitoring of respiratory muscle function in chronic respiratory diseases.
\end{abstract}

Index Terms - Non-invasive respiratory monitoring, cardiorespiratory monitoring, surface diaphragm electromyography, wearable wireless device, chronic respiratory diseases, fixed sample entropy.

\section{INTRODUCTION}

A ccording to the Organization for Economic Co-operation and Development, many countries are facing the increasing burden of an ageing population and the growing prevalence of chronic diseases [1]. In this context, new technologies must be developed to change how healthcare services are delivered in order to improve primary care systems and reduce hospital costs. A promising solution to advance the

The second author is supported by the Instituto para la Formación y Aprovechamiento de Recursos Humanos and Secretaría Nacional de Ciencia, Tecnología e Innovación (IFARHU-SENACYT Program) of the Government of Panama under grant 270-2012-273. This study was supported in part by the Spanish Ministry of Economy, Industry and Competitiveness through the project DPI2015-68820-R (MINECO/ FEDER).

M. Ràfols-de-Urquía, L. Estrada, and J. Estévez-Piorno, L. Sarlabous, R. Jané and A. Torres are with the Institut de Bioenginyeria de Catalunya (IBEC), quality of healthcare delivery is the integration of wearable wireless devices into clinical routines [2]. These technologies are designed to be small, lightweight, low cost, energy saving and easy to use, allowing physiological patient data to be monitored outside healthcare facilities without being intrusive [3], [4]. Physiological information collected with wearable wireless devices has proved beneficial in a wide range of medical applications [5] [6].

Chronic respiratory diseases, such as asthma, pulmonary hypertension, obstructive sleep apnoea and chronic obstructive pulmonary disease (COPD), compromise the airways as well as several lung structures, and are currently considered a global health issue entailing substantial healthcare costs worldwide [7]. Sixty-four million people currently suffer from COPD, and the disease is most prevalent among the elderly. It is expected to become the third leading cause of death worldwide by 2030 [8]. Bioelectrical signals, such as electrocardiography (ECG) and electromyography (EMG) can be recorded with wearable wireless devices, and together with the use of signal processing techniques, could be used to obtain important cardiorespiratory information in a cost-effective manner.

The ECG, the most traditional recorded signal originating from heart, can be recorded wirelessly by means of a disposable adhesive patch sensor worn on the chest [9]. Different information can be extracted from ECG recordings; it is not only possible to calculate heart rate (HR), but also to derive the patient's respiratory rate (RR) [10]. Other nonelectrical biomedical signals have been proposed for the extraction of multiparametric physiological information. For instance, pulse photoplethysmography can provide valuable information about the cardiovascular system when recorded by a pulse oximeter interfaced with a mobile smartphone [11], and can allow parameters such oxygen saturation, HR, RR [12] and even respiratory effort to be estimated [13].

The EMG, which represents the electrical activity generated by muscle fibres during a contraction, allows healthcare professionals to assess respiratory muscle function, providing useful information for the diagnosis and treatment of patients

the Barcelona Institute of Science and Technology (BIST). c/. Baldiri Reixac 4, 08028, Barcelona, Spain. (e-mails: \{mrafols, lestrada, jestevez, lsarlabous, rjane, atorres\}@ibecbarcelona.eu). L. Sarlabous, R. Jané and A. Torres are also with the Universitat Politècnica de Catalunya-BarcelonaTech (UPC) and the Biomedical Research Networking Center in Bioengineering, Biomaterials and Nanomedicine (CIBER-BBN). 
suffering from respiratory muscle weakness [14], such as patients with COPD. Electromyography of the diaphragm (EMGdi), the main muscle involved in the respiratory process, reflects the output of the neural respiratory centre [15]. Surface EMG of respiratory muscles, acquired by means of surface electrodes positioned at different points on the body, is a noninvasive alternative to invasive EMGdi measurements [16], [17]. Additionally, respiratory EMG signals themselves contain cardiorespiratory information from which RR and HR can be derived in neonates and adults [18], [19], [20]. These two cardiorespiratory parameters provide relevant information for evaluating patients suffering from COPD [21], [22].

Despite the potential advantages that wearable wireless devices offer in tracking health data, they have rarely been proposed for the recording of respiratory muscle activity. The present study was conducted to validate the use of a commercial wearable wireless device, the Shimmer3 (Shimmer Research Ltd., Dublin, Ireland) [24], to non-invasively record EMGdi to obtain relevant physiological data for the evaluation of cardiorespiratory function, such as respiratory muscle activity, HR and RR. The Shimmer3 is a low-cost wearable sensing platform that enables the acquisition EMG signals in unconstrained environments. However, the validity of the data acquired with the Shimmer3 for cardiorespiratory signal analysis has not been addressed. The hypothesis of this study was that cardiorespiratory measurements obtained with the Shimmer3 in healthy subjects during an inspiratory loading test in a controlled environment would be comparable to measurements made by a standard laboratory equipment. With this aim, we compare EMGdi activity recorded with a Shimmer 3 and that taken from a standard wired laboratory acquisition system in healthy subjects while performing an incremental inspiratory load protocol. Multiple types of physiological information were extracted from the Shimmer3 EMGdi signal and compared with the reference values: HR, $\mathrm{RR}$, as well as EMG activity indexes and frequency parameters to assess respiratory muscle effort.

\section{Materials AND MethodS}

\section{A. Subjects}

The following experiment was conducted with 20 nonsedentary healthy young adults (13 males and 7 females, mean \pm standard deviation: age $22.70 \pm 1.53$ years, height $1.74 \pm 0.08$ $\mathrm{m}$, weight $68.00 \pm 10.23 \mathrm{~kg}$, body mass index $22.36 \pm 1.85$ $\mathrm{kg} / \mathrm{m}^{2}$ ). The Institutional Review Board at the Institute for Bioengineering of Catalonia, Barcelona, Spain, approved all procedures (reference number IRB_IBEC_RJANE_2016_01) and participants gave their written informed consent prior to their inclusion in the study.

\section{B. Respiratory test}

Each subject underwent an incremental respiratory load test, which consisted of breathing at four different levels of inspiratory load. The subjects started by maintaining quiet breathing without the application of any additional inspiratory load. A $19 \mathrm{~cm} \mathrm{H}_{2} \mathrm{O}$ inspiratory load was applied and then raised to 29 and $41 \mathrm{~cm} \mathrm{H}_{2} \mathrm{O}$ (Load 1, Load 2 and Load 3, respectively), using a threshold inspiratory muscle trainer device (Threshold
IMT, Philips Respironics, Amsterdam, The Netherlands). The device imposes mouth pressure that requires additional inspiratory muscle effort. The respiration test during each inspiratory load lasted $60 \mathrm{~s}$ followed by a three-minute rest period. Over the course of the test, the subjects were asked to sit comfortably in a chair, maintain straight posture and rest both arms on a table, and a nose clip was provided to prevent nasal respiration. Subjects visualized their mouth pressure and airflow displayed on a screen during the test as a visual feedback to maintain a regular respiratory pattern within each inspiratory load.

\section{Data acquisition}

Two surface EMGdi signals were simultaneously recorded in this study. Each signal was recorded by means of two circular disposable $\mathrm{Ag} / \mathrm{AgCl}$ electrodes (11-mm diameter, pregelled, foam electrode 50/PK - EL501, Biopac Systems, Santa Barbara, CA, USA) in bipolar configuration, one $25 \mathrm{~mm}$ below the other. Each pair was attached to the lower right chest, on the line between the anterior axillary line and the midclavicular line, over the seventh and eighth intercostal spaces above the costal margin. This area corresponds to the zone of apposition of the costal diaphragm muscle [25]. To reach the utmost similarity between measurements, the electrodes in bipolar configuration were placed adjacent to one another as shown in Fig. 1. Prior to acquiring the data, all the electrode recording sites were carefully prepared using an abrasive cream (Nuprep, Weaver and Company, Aurora, CO, USA) and cleaned with isopropyl alcohol to improve skin/electrode impedance.

One of the EMGdi signals (EMGdiw) was acquired using a Shimmer3 (Shimmer Research Ltd., Dublin, Ireland) [24] attached with a strap to the chest of the subject. This device has a variable gain amplifier with a gain of up to 12 , a bandwidth of $8500 \mathrm{~Hz}$ and a 24-bit resolution analogue-to-digital converter. The sample rate for the acquisitions was set at 1024 $\mathrm{Hz}$, for which the Shimmer3 applies a low-pass filter with a cutoff frequency of $268.29 \mathrm{~Hz}$. The data was stored in an integrated microSD card.

The second EMGdi signal (EMGdiL) was acquired using a standard laboratory data acquisition system (MP150, Biopac System Inc., Santa Barbara, CA, USA) with a 16-bit analogueto-digital converter plugged into a modular differential amplifier (EMG100C, Biopac Systems, Inc.) with an analogue low-pass filter with a cut-off frequency of $300 \mathrm{~Hz}$ and a gain of

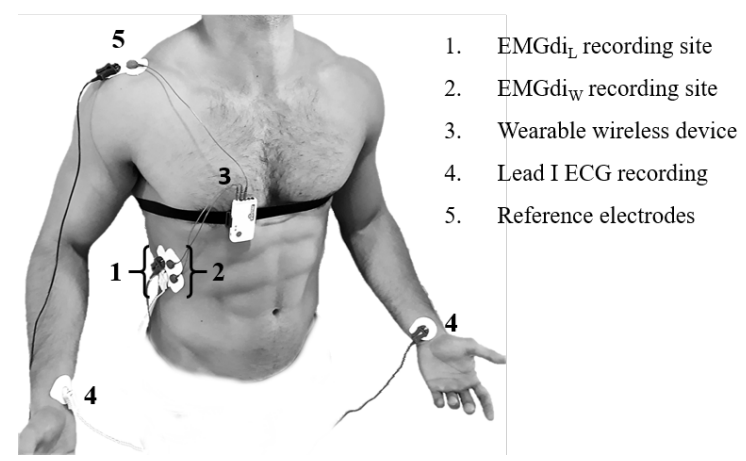

Fig. 1. Electrode set-up for signal acquisition 


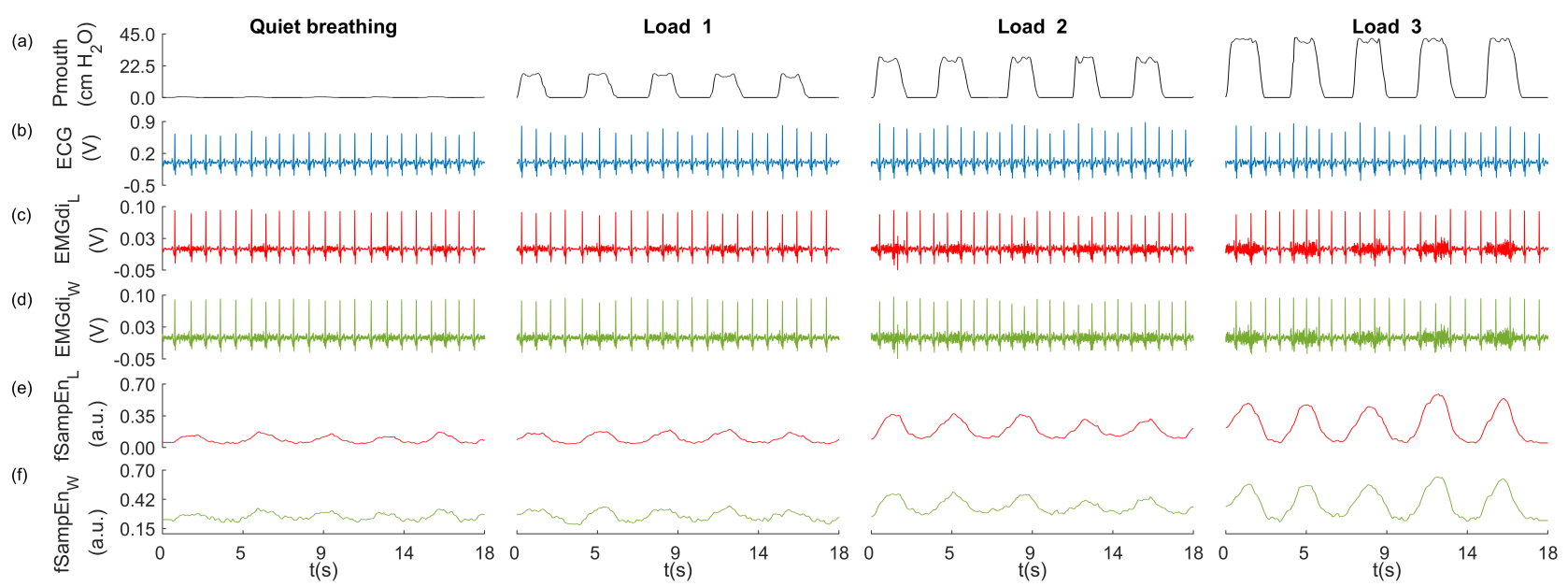

Fig. 2. Incremental inspiratory load test. For each load, representative traces during the time-course of five breathings for (a) Pmouth, (b) ECG, (c) EMGdi (d) EMGdiw, (e) fSampEnL and (f) fSampEnw. From left to right: increments in the inspiratory load corresponding to quiet breathing, and inspiration loads of 19,29 and $41 \mathrm{~cm} \mathrm{H}_{2} \mathrm{O}$.

1000. Simultaneously with the EMGdi acquisitions, the ECG and inspiratory mouth pressure (Pmouth) signals were recorded. The ECG was measured using a standard lead-I derivation with two electrodes of the same type placed on the right and left wrists and connected to an ECG modular amplifier (ECG100, Biopac Systems, Inc.) set with a gain of 1000. The Pmouth was measured using a differential pressure transducer (TSD160A, Biopac Systems, Inc.) connected to a modular amplifier (DA100C, Biopac System, Inc.). EMGdi L, ECG and Pmouth signals were recorded at $2000 \mathrm{~Hz}$, displayed in real time on a monitor screen and saved on a computer (AcqKnowledge software v.3.2 Biopac Systems Inc.).

\section{Signal processing}

The following processing steps were applied to determine this physiological information (a video with a flowchart of these processing steps is available as Supplementary Files on ScholarIOne Manuscripts):

\section{1) Signal pre-processing}

For accurate comparison with EMGdiw, the sampling frequency of the EMGdi , ECG and Pmouth signals were resampled from $2000 \mathrm{~Hz}$ to $1024 \mathrm{~Hz}$. Moreover, as the acquisitions were triggered manually, an inherent asynchrony occurred between the signals recorded with the two different acquisition systems. To resolve this issue, a technique based on the cross-correlation function was used for the temporal alignment of the signals: (1) EMGdiw and EMGdiL signals were filtered with a zero-phase fourth-order Butterworth filter with cut-off frequencies of 0.5 to $40 \mathrm{~Hz},(2)$ the normalized crosscorrelation sequence between both filtered signals at all possible lags was calculated, (3) the estimated delay is obtained as the lag for which the normalized cross-correlation has the largest absolute value, (4) all original Biopac and Shimmer3 signals were synchronized using the delay information. Afterwards, for each subject and inspiratory load, and according to Pmouth recordings, five consecutive respiratory cycles were selected where the breathing pattern was stable with no remarkably short or prolonged breaths [17], as shown in Fig. 2.

\section{2) Heart rate extraction}

EMG respiratory activity recorded on the chest is highly influenced by ECG. Taking advantage of the presence of cardiac activity, a simple QRS complex detector based on the classical Pan-Tompkins algorithm [25] was implemented for EMGdi signals. Prior to this step, EMGdic, EMGdiw and ECG signals were digitally band-pass filtered with a zero-phase fourth-order Butterworth filter with cut-off frequencies of 0.5 to $40 \mathrm{~Hz}$. Afterward, the mean HR was estimated on the selected five consecutive respiratory cycles for each load from the EMGdiw $\left(\mathrm{HR}_{\mathrm{W}}\right)$, EMGdiL $\left(\mathrm{HR}_{\mathrm{L}}\right)$ and compared to the reference heart rate (HR $\mathrm{HR}_{\mathrm{ref}}$ ) extracted from ECG by means of the same procedure applied to the EMGdi signals.

\section{3) Respiratory rate extraction}

EMGdiw and EMGdiL were digitally band-pass filtered with a zero-phase fourth-order Butterworth filter with cut-off frequencies of 5 and $400 \mathrm{~Hz}$. EMGdi activity was estimated using a fixed sample entropy (fSampEn) algorithm [23], [26]. fSampEn quantifies in short datasets, the amplitude variation of the complex components of the EMGdi while being less sensitive to deterministic components, such as ECG. A detailed explanation of the fSampEn algorithm can be found in [23]. Three parameters must be fixed in order to make use of the fSampEn: the length of compared runs $(m)$, the tolerance value (r) and the size of the moving window. In this study, we used $\mathrm{m}$ $=1$ and $\mathrm{r}=0.3$ times the standard deviation of the entire EMGdi over a moving window of $1 \mathrm{~s}$ and with $90 \%$ overlap [23], [27]. This algorithm was applied to EMGdiL and EMGdiw, leading to $\mathrm{fSampEn}_{\mathrm{L}}$ and $\mathrm{fSampEn}$, respectively.

The Pmouth, fSampEn ${ }_{L}$ and fSampEnw (Fig. 2 a, e and f, respectively) are low frequency signals directly related to breathing activity and from which RR can be extracted. To this end, firstly these signals were resampled to a new sample rate of $5 \mathrm{~Hz}$. Before resampling, all signals were low-pass filtered using a zero-phase $8^{\text {th }}$ order Chebyshev Type I filter with a cutoff frequency of $2 \mathrm{~Hz}$. Then the power spectral density was calculated via the Welch periodogram using a 10 seconds Hamming window, a 50\% window overlap and an FFT size of 4096 points. Subsequently, the mean RR on the five respiratory cycles was determined by detecting the peak frequency of the resultant spectrum. For each load, the RR was estimated from 
fSampEn $n_{L}\left(R_{L}\right)$ and fSampEnw $\left(R R_{W}\right)$, and compared to a $R R$ reference value $\left(\mathrm{RR}_{\mathrm{ref}}\right)$, obtained from the Pmouth.

4) Assessment of respiratory muscle activity

EMGdi is considered a biomarker which reflects the balance between the load on the respiratory system and the capacity of the respiratory muscles to handle that load [15]. To assess respiratory muscle activity, an EMG activity index extracted from the fSampEn was used to compare the evolution of the EMGdi signals with the level of mouth pressure applied [23]. Thus, the mean value of each respiratory cycle at each load was calculated in the two EMGdi, fSampEnL and fSampEnw (MEL and $\mathrm{MEw}$, respectively). Additionally, as Pmouth make give a reasonable approximation of the overall mechanical output of the respiratory system [14], both $\mathrm{ME}_{\mathrm{L}}$ and $\mathrm{MEw}$ were compared with the mean value of Pmouth (MP). At each load, the 5-cycle average of $\mathrm{ME}_{\mathrm{L}}, \mathrm{ME}_{\mathrm{W}}$ and $\mathrm{MP}$ values $\left(\mathrm{mME}_{\mathrm{L}}\right.$, mMEw and $\mathrm{mMP}$ ) was calculated for each subject.

\section{5) Assessment of respiratory muscle fatigue}

The analysis of the frequency content of EMGdi signals could allow muscle fatigue to be assessed [29], [30]. To appraise the power spectral density of the EMGdi signals in respiratory muscles, it was first necessary to consider only the inspiratory periods of the signal, and then to discard the segments containing QRS activity. Therefore, the preliminary stage consisted of segmenting the EMG signals to obtain segments with inspiratory activity only [28]. To extract the initial and final instant of inspiration, the algorithm compares the low-pass filtered pressure signal (using a zero-phase fourthorder Butterworth filter with a cut-off frequency of $5 \mathrm{~Hz}$ ) with a moving average of the pressure signal whose window length is adjusted in accordance with respiratory frequency. The initial and final time of cardiac activity were considered $150 \mathrm{~ms}$ before and $300 \mathrm{~ms}$ after the R point of the QRS complex, respectively. Once the EMG signals have been segmented, it is possible to accurately evaluate the power spectral density estimated in every single segment with the modified periodogram method using a Hamming window $($ NFFT $=4096)$. Then, a weighted mean periodogram was calculated with all the segments. To quantify the changes in the frequency content of EMGdiL and EMGdiw during inspiration, the mean frequency of the weighted periodogram was computed $\left(\mathrm{MF}_{\mathrm{L}}\right.$ and $\mathrm{MFw}$, respectively).

\section{E. Statistical analysis}

Friedman's non-parametric test was conducted to determine significant differences between $\mathrm{HR}$ and $\mathrm{RR}$ parameters obtained from the two acquisition systems for each inspiratory load and between ME measures across inspiratory loads. Dunn post hoc test with Bonferroni adjustment was performed for pair-wise comparisons. Wilcoxon signed-rank test was performed to compare the MF measures between the two acquisition systems for each inspiratory load. The strength of the relationship between HR, RR, and MF parameters obtained from the two acquisition systems for each inspiratory load was examined using the Pearson's correlation coefficient $(R)$. Additionally, the agreement between measurements was tested using the Bland-Altman plot analysis. Each Bland-Altman plot was constructed by plotting the differences between the data obtained from EMGdiL and EMGdiw from paired signals on the
TABLE I

POSITIVE PREDICTIVE VALUE AND SENSITIVITY OF THE HEART RATE ESTIMATION FROM THE EMGDI SIGNALS CONSIDERING THE HR ESTIMATION FROM THE ECG AS A GOLD STANDARD

\begin{tabular}{ccccc}
\hline \hline & \multicolumn{2}{c}{ PPV (\%) } & \multicolumn{2}{c}{ S (\%) } \\
\hline & $H_{W}$ & $H_{\mathrm{L}}$ & $\mathrm{HR}_{W}$ & $\mathrm{HR}_{\mathrm{L}}$ \\
\hline Quiet Breathing & 99.32 & 97.86 & 97.76 & 97.86 \\
\hline Load 1 & 94.93 & 97.04 & 98.68 & 98.68 \\
\hline Load 2 & 95.42 & 94.38 & 97.93 & 97.93 \\
\hline Load 3 & 93.03 & 93.95 & 95.87 & 94.79 \\
\hline Total & 95.67 & 95.81 & 97.56 & 97.32 \\
\hline \hline
\end{tabular}

PPV (\%): positive predictive value (TP/(TP+FP)*100), S (\%) sensitivity $(\mathrm{TP} /(\mathrm{TP}+\mathrm{FN}) * 100)$, TP: true positives, FP: false positives, $\mathrm{FN}$ : false negatives. HRw: heart rate (beats/min) from EMGdiw, HRw: heart rate (beats/min) from EMGdi,$H_{\text {ref: }}$ heart rate (beats/min) from ECG.

$y$-axis against their means on the $x$-axis. For each parameter, we calculated the mean of the differences, the standard deviation of the difference and the limits of agreement, which correspond to mean difference \pm two standard deviations.

\section{RESULTS}

All subjects completed the respiratory protocol successfully, reaching the pressure values imposed at each load level (quiet breathing: $0.38 \pm 0.24 \mathrm{~cm} \mathrm{H}_{2} \mathrm{O}$, Load 1: $20.34 \pm 2.22 \mathrm{~cm} \mathrm{H}_{2} \mathrm{O}$, Load 2: $29.69 \pm 2.29 \mathrm{~cm} \mathrm{H}_{2} \mathrm{O}$ and Load 3: $41.36 \pm 4.88 \mathrm{~cm} \mathrm{H}_{2} \mathrm{O}$, respectively). Mean HR values at each load level range from 61.86 to 72.23 beats $/ \mathrm{min}$ (quiet breathing: $61.86 \pm 9.64$ beats/min, load 1: $67.33 \pm 9.43$ beats/min, load 2: $71.38 \pm 9.59$ beats/min and load 3: $72.23 \pm 9.73$ beats/min, respectively). Mean RR values at each load level range from 13.81 to 14.47 breaths/min (quiet breathing: $14.36 \pm 4.06$ breaths $/ \mathrm{min}$, load 1: $13.81 \pm 4.75$ breaths $/ \mathrm{min}$, load 2: $14.47 \pm 5.61$ breaths $/ \mathrm{min}$ and load 3: $14.07 \pm 4.97$ breaths/min, respectively).

\section{A. Heart rate estimation}

For the estimation of HR, one subject had to be excluded because of the poor quality of the reference ECG signal, probably due to the inadequate attachment of one of the electrodes to the subject's wrist. Heart beats were successfully identified from both EMGdiL and EMGdiw systems with an overall detection rate or sensitivity of $97.56 \%$ and $97.32 \%$ and positive predictive values of $95.67 \%$ and $95.81 \%$, respectively (Table I).

Table II shows the HR values obtained from the Pmouth reference signal, EMGdiL and EMGdiw. We obtained a very strong general correlation $(R=0.989)$ between the HRw and the $H_{L}$ and no significant differences were found between $H_{L}$ and $\mathrm{HR}_{\mathrm{W}}$ for any load $(\mathrm{p}>0.05)$. The correlation between $\mathrm{HR}_{\mathrm{W}}$ and $\mathrm{HR}_{\text {ref }}$ (Fig. 3a) of overall loads was very strong $(R=0.947)$ and very similar to the correlation between $\mathrm{HR}_{\mathrm{L}}$ and $\mathrm{HR}_{\text {ref }}(R=$ 0.959). Most of the values were located between the limits of agreements and close to the bias line (Fig. 3b). As shown, an increase in the inspiratory load, and therefore EMG activity, can disturb heartbeat detection, slightly decreasing the $R$ values.

\section{B. Respiratory rate estimation}

Table III shows the RR values obtained from the Pmouth reference signal, EMGdiL and EMGdiw. A very strong correlation was found between $\mathrm{RR}_{\mathrm{L}}$ and $\mathrm{RR}_{\mathrm{W}}(R=0.989)$ at all the inspiratory load levels, as well as when $R_{L}$ and $R R_{W}$ were 
(a)

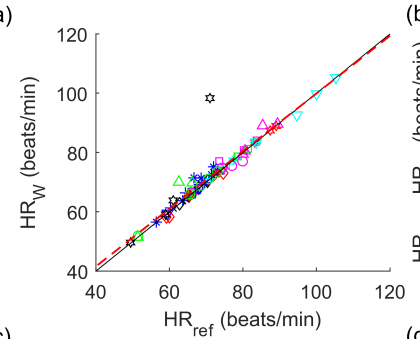

(c)

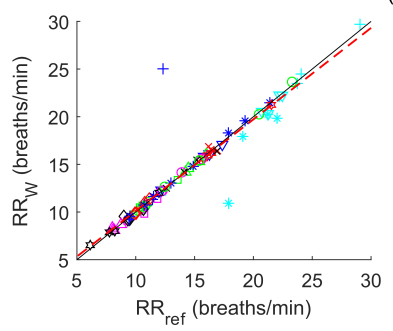

(b)

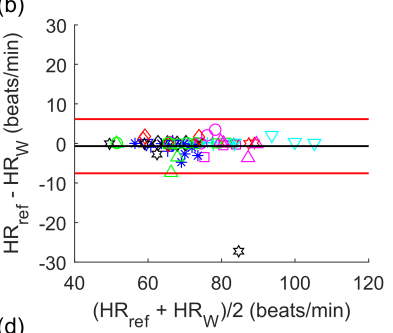

(d)

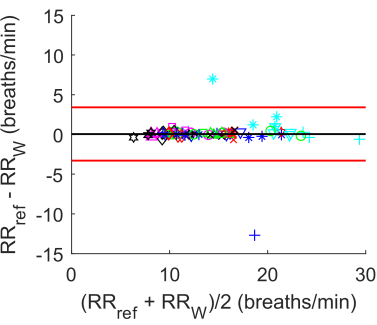

Fig. 3. Comparison of heart rate (HR) and respiratory rate (RR) obtained with a wearable wireless device and lab equipment. (a and c) Scatterplots showing the correlation between pairs of HR and RR values, respectively. The line of identity and the least square regression line are represented by a solid black line and a dashed red line, respectively. (b and d) BlandAltman plots indicating the differences between the acquisition systems for HR and RR values, respectively. The central horizontal black line depicts the mean of the differences between paired data, and the top and bottom horizontal red lines are the mean of the difference of the data with \pm 2 times the standard deviation of their differences. In all plots, each combination of symbol and colour correspond to the data from one subject ( 80 points in total). There are four elements of each symbol/colour corresponding to the four respiratory conditions (quiet breathing and inspiration with loads of 19,29 and $41 \mathrm{~cm} \mathrm{H}_{2} \mathrm{O}$ ). $\mathrm{HR}_{W}$ : heart rate (beats/min) from EMGdiw, $\mathrm{HR}_{\mathrm{L}}$ : heart rate from $E M G i_{L}, \mathrm{HR}_{\text {ref: }}$ heart rate from $E C G, R_{W}$ : respiratory rate from $E M G d_{i}, R_{L}$ : respiratory

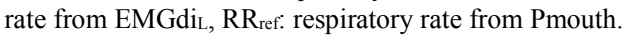

compared against the RR values extracted from the Pmouth, $(R$ $=0.951$ and $R=0.940$, respectively). The mean differences

between $R_{W}-R_{L}, R_{W}-R R_{\text {ref }}$ and $R R_{L}-R R_{\text {ref }}$ were always lower than 0.5 breaths/min.

Fig. 3c shows the correlation plot between the $\mathrm{RR}_{W}$ and the

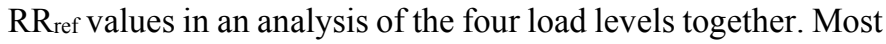
of the values were very close to the mean difference line (Fig. 3d). According to the reference value, the estimation proved inaccurate on only two occasions. When comparing $\mathrm{RR}_{\mathrm{L}}$ and $\mathrm{RR}_{\mathrm{W}}$ against $\mathrm{RR}_{\text {ref }}$ at each load independently, the $R$ value was very close to 1 when a load was applied, showing a high degree of accuracy in the respiratory cycle detection algorithm. In contrast, a lesser but still strong correlation was obtained at quiet breathing $\left(R=0.782\right.$ and $R=0.716$, for the $\mathrm{RR}_{\mathrm{L}}$ and $\mathrm{RRw}$ respectively), due to the two outliers previously mentioned.

\section{Respiratory muscle activity evaluation}

For each load, the 5-cycle average $\mathrm{mME}_{\mathrm{L}}, \mathrm{mME}_{\mathrm{W}}$ and $\mathrm{mMP}$ was calculated for each subject, the overall mean and standard deviation of which are provided in Table IV. Fig. 4 shows the box plot analysis of the $\mathrm{mME}_{\mathrm{L}}$ and $\mathrm{mME}_{\mathrm{W}}$ values obtained from the subjects. No significant differences were found between $\mathrm{mME}_{\mathrm{L}}$ and $\mathrm{mME}_{\mathrm{W}}$ for any load. Statistically significant differences $(p<0.05)$ were found when comparing the $m \mathrm{mE}_{\mathrm{L}}$ and $\mathrm{mME}_{\mathrm{W}}$ values at quiet breathing, to the respective values found when a load was applied (Load 1, Load 2 and Load 3). Finally, positive high correlations were found between the $\mathrm{MEW}$ and MP $(R=0.877)$, in concordance with those found between MEL and MP $(R=0.819)$ as shown in Table V.

\section{EMG frequency evaluation}

Table VI shows the MF values of the EMG signals, the mean difference between them, and the $R$ correlation coefficient at each load. Strong correlations were found between the mean frequency values obtained from EMGdi E $_{\text {and }}$ EMGdiw signals, at each load and when analysing all the loads together.

TABLE II.

HEART RATE, MEAN DIFFERENCE AND CORRELATION BETWEEN THE RESULTS OBTAINED FROM THE REFERENCE SIGNAL (ECG) AND THE RESULTS OBTAINED FROM THE EMGDI SIGNAL ACQUIRED BY THE LAB SYSTEM AND THE WEARABLE DEVICE

\begin{tabular}{lccccc}
\hline \hline & Quiet breathing & Load 1 & Load 2 & Load 3 & Total \\
\hline HR $_{\text {Ref }}$ & $61.86 \pm 9.64$ & $67.33 \pm 9.43$ & $71.38 \pm 9.59$ & $72.23 \pm 9.73$ & $68.20 \pm 8.98$ \\
\hline HR $_{\mathbf{L}}$ & $61.70 \pm 9.52$ & $67.64 \pm 8.67$ & $71.64 \pm 9.06$ & $73.88 \pm 9.12$ & $68.72 \pm 8.09$ \\
\hline $\mathbf{H R}_{\mathbf{W}}$ & $61.85 \pm 9.62$ & $67.50 \pm 9.33$ & $71.89 \pm 9.40$ & $74.17 \pm 10.04$ & $68.85 \pm 8.34$ \\
\hline $\mathbf{H R}_{\mathbf{L}}-\mathbf{H R}_{\mathbf{R e f}}$ & $0.170 \pm 0.654$ & $-0.326 \pm 1.884$ & $-0.274 \pm 1.439$ & $-1.741 \pm 5.243$ & $-0.543 \pm 2.928$ \\
\hline $\mathbf{H R}_{W}-\mathbf{H R}_{\mathbf{R e f}}$ & $0.012 \pm 0.339$ & $-0.182 \pm 1.326$ & $-0.537 \pm 1.263$ & $-2.039 \pm 6.540$ & $-0.687 \pm 3.428$ \\
\hline $\mathbf{H R}_{\mathbf{L}}-\mathbf{H R}_{\mathbf{W}}$ & $-0.157 \pm 0.551$ & $0.144 \pm 1.844$ & $-0.263 \pm 0.840$ & $-0.298 \pm 2.512$ & $-0.143 \pm 0.803$ \\
\hline $\boldsymbol{R}_{\text {Lvsef }}$ & 0.998 & 0.982 & 0.990 & 0.847 & 0.959 \\
\hline $\boldsymbol{R}_{\mathbf{W v R e f}}$ & 0.999 & 0.990 & 0.991 & 0.782 & 0.947 \\
\hline $\boldsymbol{R}_{\text {Lvw }}$ & 0.998 & 0.982 & 0.997 & 0.970 & 0.989 \\
\hline \hline
\end{tabular}

HR : heart rate (beats/min) from EMGdiw, HRw: heart rate (beats/min) from EMGdi, $\mathrm{HR}_{\text {ref: }}$ heart rate (beats/min) from ECG, R: Pearson's correlation coefficient. All R values were significant with $\mathrm{p}<0.01$.

TABLE III

RESPIRATORY RATE, MEAN DIFFERENCE AND CORRELATION BETWEEN THE RESULTS OBTAINED FROM THE REFERENCE SIGNAL (PRESSURE) AND THE RESULTS OBTAINED FROM THE EMGDI SIGNAL ACQUIRED BY THE LAB SYSTEM AND THE WEARABLE DEVICE.

\begin{tabular}{lccccc}
\hline \hline & Quiet breathing & Load 1 & Load 2 & Load 3 & Total \\
\hline $\mathbf{R R}_{\text {Ref }}$ & $14.36 \pm 4.06$ & $13.81 \pm 4.75$ & $14.47 \pm 5.61$ & $14.07 \pm 4.97$ & $14.17 \pm 4.34$ \\
\hline $\mathbf{R R}_{\mathbf{L}}$ & $14.87 \pm 4.74$ & $13.74 \pm 4.72$ & $14.45 \pm 5.60$ & $13.99 \pm 4.89$ & $14.26 \pm 4.25$ \\
\hline $\mathbf{R}_{\mathbf{W}}$ & $14.55 \pm 4.68$ & $13.76 \pm 4.69$ & $14.41 \pm 5.56$ & $13.97 \pm 4.91$ & $14.17 \pm 4.15$ \\
\hline $\mathbf{R}_{\mathbf{L}}-\mathbf{R R}_{\mathbf{R e f}}$ & $-0.450 \pm 2.970^{*}$ & $0.177 \pm 0.420$ & $0.042 \pm 0.264$ & $0.089 \pm 0.533$ & $-0.036 \pm 1.520$ \\
\hline $\mathbf{R}_{\mathbf{W}}-\mathbf{R}_{\mathbf{R e f}}$ & $-0.136 \pm 3.331$ & $0.160 \pm 0.462$ & $0.075 \pm 0.306$ & $0.112 \pm 0.545$ & $0.053 \pm 1.681$ \\
\hline $\mathbf{R}_{\mathbf{L}}-\mathbf{R R}_{\mathbf{W}}$ & $0.314 \pm 1.436$ & $-0.018 \pm 0.109$ & $0.033 \pm 0.147$ & $0.023 \pm 0.055$ & $0.088 \pm 0.723$ \\
\hline $\mathbf{R}_{\text {LvRef }}$ & 0.782 & 0.996 & 0.999 & 0.995 & 0.951 \\
\hline $\mathbf{R}_{\mathbf{w s R e f}}$ & 0.716 & 0.996 & 0.998 & 0.994 & 0.940 \\
\hline $\mathbf{R}_{\text {Lvw }}$ & 0.954 & 1.000 & 1.000 & 1.000 & 0.989 \\
\hline \hline
\end{tabular}

$\mathrm{RR}_{\mathrm{W}}$ : respiratory rate (breaths/min) from EMGdiw, $\mathrm{RR}_{\mathrm{L}}$ : respiratory rate (breaths/min) from EMGdi $\mathrm{L}_{\mathrm{L}}, \mathrm{RR}_{\mathrm{ref}}$ : respiratory rate $($ breaths/min) from Pmouth, $\mathrm{R}$ : Pearson's correlation coefficient. ${ }^{*}$ Indicates statistically significant differences with $\mathrm{p}<0.01$. All $\mathrm{R}$ values were significant with $\mathrm{p}<0.01$. 
TABLE IV

MEAN VALUES OF THE PRESSURE SIGNAL, FSAMPENL AND FSAMPENw

\begin{tabular}{lccccc}
\hline \hline & Quiet breathing & Load 1 & Load 2 & Load 3 & Total \\
\hline mMP & $0.25 \pm 0.14$ & $14.49 \pm 1.80$ & $21.21 \pm 1.86$ & $28.70 \pm 4.43$ & $16.16 \pm 1.85$ \\
\hline mME $_{\mathbf{L}}$ & $0.44 \pm 0.31$ & $0.80 \pm 0.33$ & $0.91 \pm 0.36$ & $1.01 \pm 0.32$ & $0.79 \pm 0.28$ \\
\hline mME $_{\mathbf{W}}$ & $0.42 \pm 0.21$ & $0.75 \pm 0.29$ & $0.84 \pm 0.31$ & $0.92 \pm 0.30$ & $0.73 \pm 0.22$ \\
\hline \hline
\end{tabular}

mMP: average of the mean pressure in each cycle $\left(\mathrm{cm} \mathrm{H}_{2} \mathrm{O}\right)$, mME $\mathrm{E}_{\mathrm{L}}$ average of the mean entropy in each cycle of the $\mathrm{fSampEn}_{\mathrm{L}}(\mathrm{a} . \mathrm{u}$.), mMEw: average of mean entropy in each cycle of the fSampEnw (a.u.).

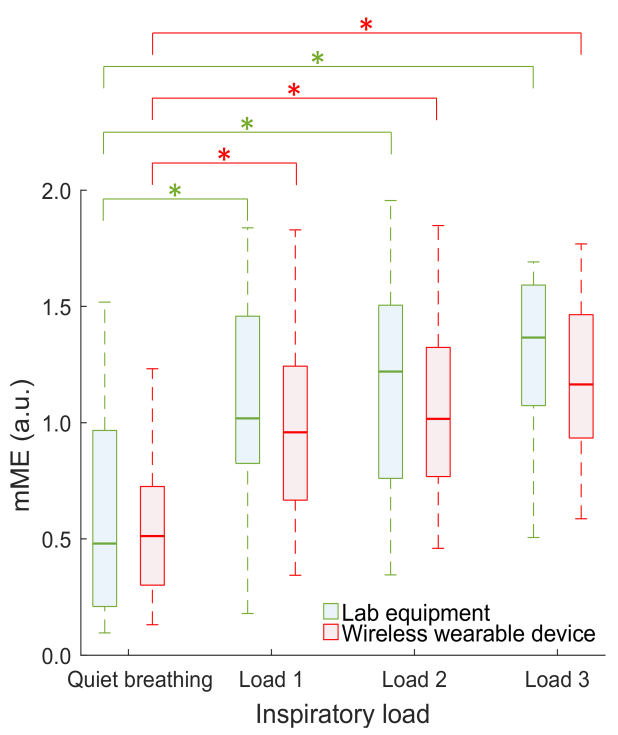

Fig. 4. Box plot comparing average of the mean amplitude in each cycle (mME) derived from $\mathrm{SSampEn}_{\mathrm{L}}$ and $\mathrm{fSampEn}_{\mathrm{W}}$ extracted from EMGdi signals recorded using the wearable device (EMGdiw) and the lab system (EMGdiL) during an incremental inspiratory load test. For each boxplot, the horizontal line represents the median, the length of the box represents the interquartile range (IQR: 25 th to 75 th percentiles), the vertical dashed lines are the whiskers and represent the largest and smallest values within 1.5 IQRs. * Indicates statistically significant differences with $p<0.01$.
TABLE V

PEARSON'S CORRELATION COEFFICIENT VALUES (R) OF EACH SUBJECT BETWEEN THE MEAN VALUES OF PMOUTH (MP) AND THE EMG ACTIVITY INDEXES OBTAINED WITH A LAB EQUIPMENT (MEL) AND THE SHIMMER3 DEVICE (MEw).

\begin{tabular}{|c|c|c|c|}
\hline Subject & 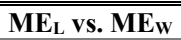 & 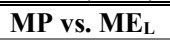 & MP vs. MEw \\
\hline 1 & 0.981 & 0.851 & 0.903 \\
\hline 2 & 0.967 & 0.928 & 0.970 \\
\hline 3 & 0.994 & 0.940 & 0.942 \\
\hline 4 & 0.985 & 0.840 & 0.883 \\
\hline 5 & 0.934 & 0.549 & 0.687 \\
\hline 6 & 0.943 & 0.886 & 0.864 \\
\hline 7 & 0.998 & 0.933 & 0.930 \\
\hline 8 & 0.998 & 0.775 & 0.749 \\
\hline 9 & 0.999 & 0.961 & 0.961 \\
\hline 10 & 0.751 & 0.741 & 0.267 \\
\hline 11 & 0.990 & 0.750 & 0.782 \\
\hline 12 & 0.994 & 0.867 & 0.863 \\
\hline 13 & 0.967 & 0.601 & 0.424 \\
\hline 14 & 0.999 & 0.829 & 0.840 \\
\hline 15 & 0.990 & 0.879 & 0.857 \\
\hline 16 & 0.989 & 0.907 & 0.928 \\
\hline 17 & 0.972 & 0.764 & 0.736 \\
\hline 18 & 0.984 & 0.980 & 0.985 \\
\hline 19 & 0.984 & 0.924 & 0.906 \\
\hline 20 & 0.995 & 0.942 & 0.933 \\
\hline Total & 0.990 & 0.819 & 0.877 \\
\hline
\end{tabular}

MP: mean amplitude of the Pmouth $\left(\mathrm{cm} \mathrm{H}_{2} \mathrm{O}\right), \mathrm{ME}_{\mathrm{L}}$ : mean entropy in each cycle of the $\mathrm{SSampEn}_{\mathrm{L}}$ (a.u.), MEw: mean entropy in each cycle of the fSampEnw (a.u.), $R$ : Pearson's correlation coefficient. All $R$ values were significant with $p<0.01$.

TABLE VI

FREQUENCY PARAMETERS OF EMG THE INSPIRATION AND COMPARISON BETWEEN THE RESULTS OBTAINED FROM EMGDIL AND EMGDIw.

\begin{tabular}{lccccc}
\hline \hline & Quiet breathing & Load 1 & Load 2 & Load 3 & Total \\
\hline $\mathbf{M F}_{\mathbf{L}}$ & $52.19 \pm 13.06$ & $55.03 \pm 14.93$ & $56.27 \pm 15.46$ & $53.77 \pm 14.81$ & $54.31 \pm 11.80$ \\
\hline $\mathbf{M F}_{\mathbf{w}}$ & $55.70 \pm 16.93$ & $56.69 \pm 15.36$ & $56.89 \pm 14.75$ & $55.23 \pm 16.98$ & $56.13 \pm 13.07$ \\
\hline $\mathbf{M F}_{\mathbf{L}}-\mathbf{M F}_{\mathbf{w}}$ & $-3.505 \pm 7.639$ & $-1.662 \pm 7.220$ & $-0.624 \pm 6.209$ & $-1.459 \pm 7.155$ & $-1.420 \pm 5.740$ \\
\hline $\boldsymbol{R}_{\mathbf{M F L v M F W}}$ & 0.902 & 0.887 & 0.917 & 0.908 & 0.895 \\
\hline \hline
\end{tabular}

$\mathrm{MF}_{\mathrm{w}}$ : mean frequency $(\mathrm{Hz})$ of the EMGdiw, $\mathrm{MF}_{\mathrm{L}}$ : mean frequency $(\mathrm{Hz})$ of the EMGdi,$R$ : Pearson's correlation coefficient. All $R$ values were significant with $p<0.01$.

\section{DISCUSSION AND CONCLUSION}

Rapid advances in technology have led to the inclusion of wearable wireless devices in medicine, contributing portability to the monitoring of physiological signals. The present study was conducted to validate the use of a Shimmer3 wearable wireless device [24] for the non-invasive recording of muscle respiratory activity outside a hospital environment and for estimating multiple cardiorespiratory parameters from a single bioelectrical signal. The parameters obtained from the EMGdi signal acquired by the Shimmer3 strongly correlated with the parameters obtained from the reference signals recorded by the standard wired laboratory acquisition system.

The acquisition system proposed in the present study, in conjunction with the signal processing algorithms designed, could accurately detect almost all HR from an EMGdi signal.
Only on one occasion at the highest load was the HR out of the limits of agreement when compared with the reference HR value (Fig. $3 \mathrm{a}$ and b). In effect, when high load is applied more muscle activity is reflected in the EMGdi in a higher amplitude and frequency, which makes it easier to miss a true beat or detect a false beat. This inaccuracy is reflected in the $R$ coefficients, S and PPV values found in Table I and Table II, respectively, which are always lower at high loads. Furthermore, this behaviour was not only present in the measurements extracted from the signals acquired by the wearable system, but also in the signals extracted by the standard laboratory acquisition system, suggesting that these differences are due to the signal conditions and not to the sensing system. Indeed, the correlation between $\mathrm{HR}_{\mathrm{L}}$ and $\mathrm{HRw}$ was very high $(R=0.989)$, independently of the load applied. 
For RR estimation we adopted the use of the fSampEn technique [31]. The cycles were successfully detected in most subjects, as a very strong general correlation was found between the $\mathrm{RR}_{\mathrm{L}}$ and $\mathrm{RR}_{\mathrm{w}}$ and the $\mathrm{RR}_{\text {ref }}(R=0.951$ and $R=$ 0.940 , respectively). As expected, correlation between the reference method and the agreement between and $\mathrm{RRW}_{\mathrm{W}}$ improved noticeably when a high load was applied. At high loads the EMGdi amplitude is high compared to the signal baseline, but at quiet breathing it is low, and therefore, difficult to detect [23]. We found a few outliers in the Bland-Altman plot, which correspond to the two low-quality fSampEn estimations in two of the subjects. A closer analysis of the fSampEn revealed that there was considerable expiratory activity in one case. This behaviour led to the false respiratory cycle detection in this subject during quiet breathing, doubling the $\mathrm{RR}_{\mathrm{L}}$ and $\mathrm{RR}_{\mathrm{W}}$ and thus, biasing the overall $R$ correlation value. This inherent condition occurred in both Shimmer3 and standard laboratory acquisition system, demonstrating that both were capturing this expiratory activity. In the other case, an unexpected low respiratory frequency was estimated during quiet breathing from the EMGdiw due to a very weak inspiratory EMG activity.

Analysing the EMGdi signal allows us not only to derive cardiorespiratory information, such as $\mathrm{HR}$ and $\mathrm{RR}$, but also to explore the respiratory muscular function and measure the neural respiratory drive which reflects the load on the respiratory muscles [15]. In the present study, we conducted an incremental load test using a threshold trainer device to impose different levels of inspiratory loads to increase diaphragm muscular effort [32]. As the values in Table IV and pictured in Fig. 4 show, the amplitude of the fSampEn $n_{L}$ and fSampEn increased when the imposed load increased. The trend was observed in EMGdiL and EMGdiw, and reflected in the fSampEn $n_{L}$ and $f S a m p E n_{W}$, as shown in Fig. 2. This behaviour, which represents the muscle effort to maintain the airflow inspired during each respiratory cycle [15], strongly correlates with the mouth pressure applied, as reported in Table V. Although all correlations were positive, there was a variability in $R$ coefficients between subjects. Indeed, to overcome absolute load levels, different subjects have to exert different muscle forces meaning different neural outputs and efforts. Moreover, the increase between the loads is not very substantial, and therefore, for several subjects, the difference between the effort needed to overcome the different loads was barely noticeable. This issue is reflected in the statistical results, as significant differences were only found between the amplitude of the signals at quiet breathing and with applied loads.

Our frequency results suggest that the study of the changes in the power spectral density of the EMGdi signal could help to detect when muscle fatigue occurs and could be used in COPD patients or in the context of respiratory muscle rehabilitation [29], [30]. In this study, the mean frequencies of the spectrum obtained with the Shimmer3 and the standard laboratory acquisition system strongly correlate, which indicates that fatigue tests could also be performed with the Shimmer3.

The present study has been carried out in twenty healthy subjects in resting conditions and selecting five consecutive respiratory cycles in each of the four inspiratory loads. Further studies using more subjects, longer recordings and different settings, with subjects performing different daily routine physical activities, should be conducted to assess if the results obtained in the present studied can be generalized to a larger population and several scenarios.

In conclusion, the use of a Shimmer3 connected to a pair of electrodes to record EMGdi activity may be an attractive alternative to wirelessly and non-invasively extract multiple cardiorespiratory parameters from one signal. The comparison between the data obtained from the EMGdi signals recorded by the two acquisition systems revealed similarities in the data collected, supporting that wearable wireless devices are capable of monitoring various cardiorespiratory parameters. These systems seem to be a promising low-cost approach for the evaluation of respiratory muscle function in free-living situations, to either measure disease severity, evolution or responses to treatment for chronic respiratory diseases.

\section{REFERENCES}

[1] OECD, Health at a Glance: Europe 2016. 2016.

[2] G. Appelboom, E. Camacho, M. E. Abraham, S. S. Bruce, E. L. Dumont, B. E. Zacharia, R. D’Amico, J. Slomian, J. Y. Reginster, O. Bruyère, and E. S. Connolly, "Smart wearable body sensors for patient self-assessment and monitoring," Arch. public Heal., vol. 72, no. 1, p. 28, Aug. 2014.

[3] Y. L. Zheng, X. R. Ding, C. C. Y. Poon, B. P. L. Lo, H. Zhang, X. L. Zhou, G. Z. Yang, N. Zhao, and Y. T. Zhang, "Unobtrusive sensing and wearable devices for health informatics," IEEE Trans. Biomed. Eng., vol. 61 , no. 5, pp. 1538-1554, May 2014.

[4] S. C. Mukhopadhyay, "Wearable sensors for human activity monitoring: A review," IEEE Sens. J., vol. 15, no. 3, pp. 1321-1330, Mar. 2014.

[5] A. Pantelopoulos and N. G. Bourbakis, "A survey on wearable sensorbased systems for health monitoring and prognosis," vol. 40, no. 1, pp. 1-12, Jan. 2010.

[6] S. Patel, H. Park, P. Bonato, L. Chan, and M. Rodgers, "A review of wearable sensors and systems with application in rehabilitation," $J$. Neuroeng. Rehabil., vol. 9, no. 1, p. 21, Apr. 2012.

[7] N. Aitt-Khaled, D. Enarson, and J. Bousquet, "Chronic respiratory diseases in developing countries: the burden and strategies for prevention and management," Bull. World Health Organ., vol. 79, no. 10, pp. 971-979, 2001.

[8] World Health Organization, "Burden of COPD," 2011. [Online]. Available: http://www.who.int/entity/respiratory/copd/en/. [Accessed: 15-Nov-2017].

[9] N. Selvaraj, "Long-term remote monitoring of vital signs using a wireless patch sensor," in Healthcare Innovation Conference (HIC), 2014 IEEE, 2014, pp. 83-86.

[10] J. Boyle, N. Bidargaddi, A. Sarela, and M. Karunanithi, "Automatic detection of respiration rate from ambulatory single-lead ECG," IEEE Trans. Inf. Technol. Biomed., vol. 13, no. 6, pp. 890-896, Nov. 2009.

[11] A. Garde, P. Dehkordi, W. Karlen, D. Wensley, J. M. Ansermino, and G. A. Dumont, "Development of a screening tool for sleep disordered breathing in children using the phone oximeterTM," PLoS One, vol. 9, no. 11, p. e112959, Nov. 2014.

[12] E. Gil, M. Orini, R. Bailón, J. M. Vergara, L. Mainardi, and P. Laguna, "Photoplethysmography pulse rate variability as a surrogate measurement of heart rate variability during non-stationary conditions," Physiol. Meas., vol. 31, no. 9, pp. 1271-1290, Aug. 2010.

[13] P. S. Addison, "Respiratory effort from the photoplethysmogram," Med. Eng. Phys., vol. 41, pp. 9-18, Mar. 2017.

[14] "ATS/ERS statement on respiratory muscle testing," Am. J. Respir. Crit. Care Med., vol. 166, no. 4, pp. 518-624, Aug. 2002.

[15] P. B. Murphy, A. Kumar, C. Reilly, C. Jolley, S. Walterspacher, F. Fedele, N. S. Hopkinson, W. D.-C. Man, M. I. Polkey, J. Moxham, and N. Hart, "Neural respiratory drive as a physiological biomarker to monitor change during acute exacerbations of COPD," Thorax, vol. 66, no. 7, pp. 602-8, Jul. 2011.

[16] E. Maarsingh, L. A. Van Eykern, A. B. Sprikkelman, M. O. Hoekstra, and W. M. C. van Aalderen, "Respiratory muscle activit measured with a noninvasive EMG technique: technical aspects and reproducibility," $J$. 
Appl. Physiol., vol. 88, no. 6, pp. 1955-1961, Jun. 2000.

[17] M. L. Duiverman, L. a van Eykern, P. W. Vennik, G. H. Koëter, E. J. W. Maarsingh, and P. J. Wijkstra, "Reproducibility and responsiveness of a noninvasive EMG technique of the respiratory muscles in COPD patients and in healthy subjects," J. Appl. Physiol., vol. 96, no. 5, pp. 1723-1729, 2004.

[18] J. V Kraaijenga, G. J. Hutten, F. H. de Jongh, and A. H. van Kaam, "Transcutaneous electromyography of the diaphragm: A cardiorespiratory monitor for preterm infants," Pediatr. Pulmonol., vol. 50, no. 9, pp. 889-895, Sep. 2015.

[19] R. Torres, S. López-isaza, E. Mejía-mejía, V. Paniagua, and V. González, "Low-power system for the acquisition of the respiratory signal of neonates using diaphragmatic electromyography," Med. Devices Evid. Res., pp. 47-52, Feb. 2017.

[20] L. Estrada, A. Torres, L. Sarlabous, and R. Jané, "Evaluating respiratory muscle activity using a wireless sensor platform," in Proc 38 th Annu Int Conf IEEE EMBS, 2016.

[21] A. M. Yañez, D. Guerrero, R. Pérez De Alejo, F. Garcia-Rio, J. L. Alvarez-Sala, M. Calle-Rubio, R. Malo De Molina, M. Valle Falcones, P. Ussetti, J. Sauleda, E. Zamora García, J. M. Rodríguez-GonzálezMoro, M. Franco Gay, M. Torrent, and A. Agustí, "Monitoring breathing rate at home allows early identification of COPD exacerbations," Chest, vol. 142, no. 6, pp. 1524-1529, Dec. 2012.

[22] M. T. Jensen, J. L. Marott, P. Lange, J. Vestbo, P. Schnohr, O. W. Nielsen, J. S. Jensen, and G. B. Jensen, "Resting heart rate is a predictor of mortality in COPD," Eur. Respir. J., vol. 42, no. 2, pp. 341-349, Aug. 2013.

[23] L. Estrada, A. Torres, L. Sarlabous, and R. Jané, "Improvement in neural respiratory drive estimation from diaphragm electromyographic signals using fixed sample entropy," IEEE J. Biomed. Heal. Informatics, vol. 20, no. 2, pp. 476-485, Mar. 2016

[24] A. Burns, B. R. Greene, M. J. McGrath, T. J. O'Shea, B. Kuris, S. M. Ayer, F. Stroiescu, and V. Cionca, "SHIMMER ${ }^{\mathrm{TM}}-\mathrm{A}$ wireless sensor platform for noninvasive biomedical research," IEEE Sens. J., vol. 10, no. 9, pp. 1527-1534, Sep. 2010.

[25] J. Pan and W. J. Tompkins, "A real-time QRS detection algorithm," IEEE Trans. Biomed. Eng., vol. 32, no. 3, pp. 230-236, Mar. 1985.

[26] L. Sarlabous, A. Torres, J. A. Fiz, and R. Jané, "Evidence towards improved estimation of respiratory muscle effort from diaphragm mechanomyographic signals with cardiac vibration interference using sample entropy with fixed tolerance values," PLoS One, vol. 9, no. 2, p. e88902, Jan. 2014.

[27] L. Estrada, A. Torres, L. Sarlabous, and R. Jané, "Influence of parameter selection in fixed sample entropy of surface diaphragm

electromyography for estimating respiratory activity," Entropy, vol. 19 , no. 9 , p. 460 , Sep. 2017

[28] C. A. Sinderby, L. Lindström, N. Comtois, and A. Grassino, "Effects of diaphragm shortening on the mean action potential conduction velocity in canines," J. Physiol., vol. 490(Pt 1), pp. 207-214, Jan. 1996.

[29] M. González-Izal, A. Malanda, E. Gorostiaga, and M. Izquierdo, "Electromyographic models to assess muscle fatigue," J. Electromyogr. Kinesiol., vol. 22, no. 4, pp. 501-512, Aug. 2012.

[30] M. Cifrek, V. Medved, S. Tonković, and S. Ostojić, "Surface EMG based muscle fatigue evaluation in biomechanics," Clin. Biomech., vol. 24, no. 4, pp. 327-340, May 2009.

[31] L. Estrada, A. Torres, L. Sarlabous, and R. Jané, "Onset and offset detection of the neural respiratory activity in surface diaphragm electromyography using fixed sample Entropy: a pilot study in healthy subjects," IEEE J. Biomed. Heal. informatics, vol. 22, no. 1, pp. 67-76, Jan. 2018.

[32] A. Dornelas De Andrade, T. N. S. Silva, H. Vasconcelos, M. Marcelino, M. G. Rodrigues-Machado, V. C. Galindo Filho, N. H. Moraes, P. E. M. Marinho, and C. F. Amorim, "Inspiratory muscular activation during threshold $\AA$ therapy in elderly healthy and patients with COPD," $J$. Electromyogr. Kinesiol., vol. 15, no. 6, pp. 631-639, Dec. 2005.

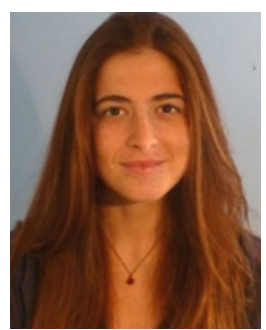

Magda Ràfols de Urquía was born in Barcelona, Spain, in 1994. She received an B.Sc. degree in biomedical engineering from the Universitat Politècnica de Catalunya (UPC) in 2015 and an M. Sc. degree in biomedical engineering from Universitat de Barcelona (UB) in conjunction with UPC in 2016. She worked as a research assistant at the Institute for Bioengineering of Catalonia (IBEC), the Barcelona Institute of Science and Technology (BIST).

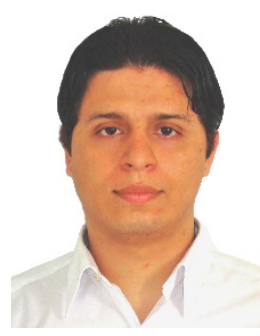

Luis Estrada (M'08, SM'17) was born in Panama City, Panama, in 1982. He received a B.Sc. degree in biomedical engineering from the Universidad Latina de Panamá, Panama, in 2004, an M.Sc. degree in biomedical engineering from the Universitat de Barcelona and the Universitat Politècnica de Catalunya (UPC) BarcelonaTech in 2009, and a Ph.D. in biomedical engineering from UPC-BarcelonaTech, Barcelona, in 2016. He is currently a postdoctoral researcher at the Institute for Bioengineering of Catalonia (IBEC), the Barcelona Institute of Science and Technology (BIST), Barcelona

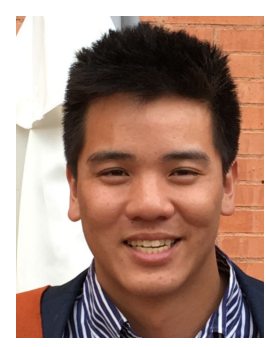

Josep Estévez Piorno was born in Barcelona, Spain, in 1992. He received an B.Sc. degree in biomedical engineering from the Universitat Politècnica de Catalunya (UPC) in 2015 and an M.Sc. degree in biomedical engineering from the Universitat de Barcelona (UB) in conjunction with UPC in 2016. He worked as a research assistant at the Institute for Bioengineering of Catalonia (IBEC), the Barcelona Institute of Science and Technology (BIST), Barcelona.

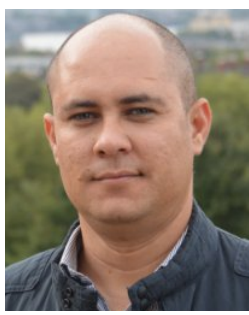

Leonardo Sarlabous was born in Santiago de Cuba, Cuba, in 1980. He earned an undergraduate degree in telecommunications and electronic engineering from the University of Oriente (Cuba) in 2005. He received his $\mathrm{Ph}$.D. in biomedical engineering from the Universitat Politècnica de Catalunya (UPC), BarcelonaTech, Spain, in 2013. He is currently a postdoctoral researcher at the Institute for Bioengineering of Catalonia (IBEC), the Barcelona Institute of Science and Technology (BIST) and an assistant professor of automatic control in the Department of Control Engineering at the UPC. $\mathrm{He}$ is also involved as a researcher at the Biomedical Research Networking Center in Bioengineering, Biomaterials and Nanomedicine (CIBER-BBN) 


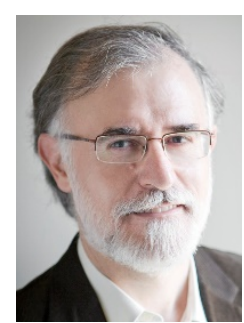

Raimon Jané (M'91, SM'14) received a Ph.D. from the Universitat Politècnica de Catalunya (UPC), Barcelona, Spain, in 1989. He is currently director of research in the Department of Automatic Control (ESAII), UPC, and the scientific group leader of the Biomedical Signal Processing and Interpretation Group, Institute for Bioengineering of Catalonia (IBEC), the Barcelona Institute of Science and Technology (BIST), Barcelona. Since 2008, he has been the principal investigator for the Biomedical Signals and Systems (SISBIO) Group of the Biomedical Research Networking Center in Bioengineering, Biomaterials and Nanomedicine (CIBER-BBN). He is a professor in the master's degree programme and the coordinator of the Ph.D. programme in biomedical engineering. In 2005, he received the Barcelona City Prize from the Barcelona City Council in technology research. Prof. Jané has been the president of the Spanish Society of Biomedical Engineering (SEIB) since 2012.

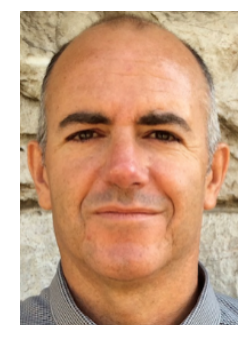

Abel Torres (M'97) was born in Figueres (Girona), Spain, in 1972. He received his M.Sc. in electrical engineering and his Ph.D. in biomedical engineering from the Universitat Politècnica de Catalunya (UPC), Barcelona, Spain, in 1997 and 2003, respectively. From 1998 to 2002, he worked as a research fellow at the Biomedical Engineering Research Centre (CREB) in Barcelona, Spain. He is currently a professor of automatic control in the Department of Control Engineering at UPC and he is involved as a senior researcher with the Institute for Bioengineering of Catalonia (IBEC), the Barcelona Institute of Science and Technology (BIST) and the Biomedical Research Networking Center in Bioengineering, Biomaterials and Nanomedicine (CIBER-BBN). 